\title{
ON CERTAIN IDENTITY RELATED TO JORDAN *-DERIVATIONS
}

\author{
IRENA Kosi-UlBL AND Joso VuKMAN \\ University of Maribor, Slovenia
}

\begin{abstract}
In this paper we prove the following result. Let $H$ be a real or complex Hilbert space, let $\mathcal{L}(H)$ be the algebra of all bounded linear operators on $H$ and let $A(H) \subseteq \mathcal{L}(H)$ be a standard operator algebra. Suppose we have an additive mapping $D: A(H) \rightarrow \mathcal{L}(H)$ satisfying the relation $D\left(A^{n}\right)=D(A) A^{* n-1}+A D\left(A^{n-2}\right) A^{*}+A^{n-1} D(A)$ for all $A \in$ $A(H)$ and some fixed integer $n>1$. In this case there exists a unique $B \in \mathcal{L}(H)$ such that $D(A)=B A^{*}-A B$ holds for all $A \in A(H)$.
\end{abstract}

Throughout, $R$ will represent an associative ring with center $Z(R)$. Given an integer $n \geq 2$, a ring $R$ is said to be $n$-torsion free if for $x \in R, n x=0$ implies $x=0$. An additive mapping $x \mapsto x^{*}$ on a ring $R$ is called an involution if $(x y)^{*}=y^{*} x^{*}$ and $x^{* *}=x$ hold for all $x, y \in R$. A ring equipped with an involution is called a ring with involution or ${ }^{*}$-ring. Recall that a ring $R$ is prime if for $a, b \in R, a R b=(0)$ implies that either $a=0$ or $b=0$, and is semiprime in case $a R a=(0)$ implies $a=0$. An additive mapping $D: R \rightarrow R$, where $R$ is an arbitrary ${ }^{*}$-ring is called a ${ }^{*}$-derivation in case $D(x y)=D(x) y^{*}+x D(y)$ holds for all pairs $x, y \in R$ and is called a Jordan *-derivation if $D\left(x^{2}\right)=D(x) x^{*}+x D(x)$ is fulfilled for all $x \in R$. It is easy to prove that there are no nonzero ${ }^{*}$-derivations on noncommutative prime ${ }^{*}$-rings (see [1] for the details). Note that the mapping $x \mapsto a x^{*}-x a$, where $a \in R$ is a fixed element, is a Jordan *-derivation; such Jordan *-derivations are said to be inner. By our knowledge the concept of Jordan *-derivations first appeared in [1]. The study of Jordan *-derivations has been motivated by the problem of the representability of quadratic forms by bilinear forms (for the results concerning this problem we refer to [6-10,13,14,16-19,22,23]). It turns

2010 Mathematics Subject Classification. 16W10, 46K15, $39 \mathrm{~B} 05$.

Key words and phrases. Ring, ring with involution, prime ring, semiprime ring, Hilbert space, standard operator algebra, ${ }^{*}$-derivation, Jordan ${ }^{*}$-derivation. 
out that the problem whether each quadratic form can be represented by some bilinear form is closely connected with the structure of Jordan *-derivations $([2,15])$. In [1] Brešar and the second named author of the present paper studied some algebraic properties of Jordan *-derivations. As a special case of Theorem 1 in [1] we have that every Jordan *-derivation of a complex algebra $A$ with the identity element is inner. Let $X$ be a real or complex Banach space, and let $\mathcal{L}(X)$ and $\mathcal{F}(X)$ denote the algebra of all bounded linear operators on $X$ and the ideal of all finite rank operators in $\mathcal{L}(X)$, respectively. An algebra $A(X) \subseteq \mathcal{L}(X)$ is said to be standard in case $\mathcal{F}(X) \subset A(X)$. In case $X$ is a Hilbert space we denote by $A^{*}$ the adjoint operator of $A \in \mathcal{L}(X)$.

We start with the following result proved by Šemrl $([15])$.

THEOREM 1. Let $H$ be a real or complex Hilbert space and let $A(H)$ be a standard operator algebra. Suppose that $D: A(H) \rightarrow \mathcal{L}(H)$ is an additive mapping satisfying the relation

$$
D\left(A^{2}\right)=D(A) A^{*}+A D(A)
$$

for all $A \in A(H)$. In this case there exists a unique $B \in \mathcal{L}(H)$ such that $D(A)=B A^{*}-A B$ holds for all $A \in A(H)$.

In case $D: R \rightarrow R$ is a Jordan *-derivation, where $R$ is an arbitrary *-ring, one can easily prove that

$$
D(x y x)=D(x) y^{*} x^{*}+x D(y) x^{*}+x y D(x),
$$

holds for all pairs $x, y \in R$. The above relation has been considered in $[5,11,23]$. It seems natural to ask under what additional assumptions an additive mapping $D$ satisfying the relation (1) is a Jordan *-derivation. The second named author of the present paper [20] has proved the following result.

TheOREM 2. Let $R$ be a 6-torsion free semiprime ${ }^{*}$-ring, and let $D: R \rightarrow$ $R$ be an additive mapping satisfying the relation (1) for all $x \in R$. In this case $D$ is a Jordan *-derivation.

Putting $x^{n-2}$ for $y$ in the relation (1), where $n>1$ is some fixed integer, one obtains the relation bellow

$$
D\left(x^{n}\right)=D(x) x^{* n-1}+x D\left(x^{n-2}\right) x^{*}+x^{n-1} D(x), \quad x \in R .
$$

In case $n=3$ the relation above reduces to the special case of the relation which has been considered in [21]. It is our aim in this paper to prove the result below, which is related to the equation (2).

TheOREM 3. Let $H$ be a real or complex Hilbert space and let $A(H)$ be a standard operator algebra. Suppose we have an additive mapping $D: A(H) \rightarrow$ $\mathcal{L}(H)$ satisfying the relation

$$
D\left(A^{n}\right)=D(A) A^{* n-1}+A D\left(A^{n-2}\right) A^{*}+A^{n-1} D(A)
$$


for all $A \in A(H)$ and some fixed integer $n>1$. In this case there exists a unique $B \in \mathcal{L}(H)$ such that $D(A)=B A^{*}-A B$ holds for all $A \in A(H)$.

Let us point out that in the theorem above we obtain as a result the continuity of $D$ under purely algebraic requirements concerning $D$, which means that the result above might be of some interest from the automatic continuity point of view. For results concerning automatic continuity we refer to $[3,4,12]$.

Proof of Theorem 3. We have the relation

$$
D\left(A^{n}\right)=D(A) A^{* n-1}+A D\left(A^{n-2}\right) A^{*}+A^{n-1} D(A) .
$$

Let $A$ be from $\mathcal{F}(H)$ and let $P \in \mathcal{F}(H)$ be a self-adjoint projection with $A P=P A=A$. Of course, we have also $A^{*} P=P A^{*}=A^{*}$. From the relation (3) one obtains

$$
D(P)=D(P) P+P D(P) P+P D(P) .
$$

Right multiplication of the above relation by $P$ gives

$$
P D(P) P=0 \text {. }
$$

Multiplying the relation (4) from the left side by $A$ and from the right side by $A^{*}$ we obtain

$$
A D(P) A^{*}=0 .
$$

Putting $A+P$ for $A$ in the relation (3), we obtain

(6)

$$
\begin{aligned}
& \sum_{i=0}^{n}\left(\begin{array}{c}
n \\
i
\end{array}\right) D\left(A^{n-i} P^{i}\right) \\
&= D(A+P)\left(\sum_{i=0}^{n-1}\left(\begin{array}{c}
n-1 \\
i
\end{array}\right) A^{* n-1-i} P^{i}\right) \\
&+(A+P)\left[\begin{array}{l}
n-2 \\
i=0
\end{array}\left(\begin{array}{c}
n-2 \\
i
\end{array}\right) D\left(A^{* n-2-i} P^{i}\right)\right]\left(A^{*}+P\right) \\
&+\left(\sum_{i=0}^{n-1}\left(\begin{array}{c}
n-1 \\
i
\end{array}\right) A^{n-1-i} P^{i}\right) D(A+P) .
\end{aligned}
$$

Using (3) and rearranging the equation (6) in sense of collecting together terms involving equal number of factors of $P$ we obtain:

$$
\sum_{i=1}^{n-1} f_{i}(A, P)=0
$$

where $f_{i}(A, P)$ stands for the expression of terms involving $i$ factors of $P$. Replacing $A$ by $A+2 P, A+3 P, \ldots, A+(n-1) P$ in turn in the equation 
(3), and expressing the resulting system of $n-1$ homogeneous equations of variables $f_{i}(A, P), i=1,2, \ldots, n-1$, we see that the coefficient matrix of the system is a van der Monde matrix

$$
\left[\begin{array}{cccc}
1 & 1 & \cdots & 1 \\
2 & 2^{2} & \cdots & 2^{n-1} \\
\vdots & \vdots & \vdots & \vdots \\
n-1 & (n-1)^{2} & \cdots & (n-1)^{n-1}
\end{array}\right] .
$$

Since the determinant of the matrix is different from zero, it follows that the system has only a trivial solution. In particular,

$$
\begin{aligned}
f_{n-1}(A, P)= & \left(\begin{array}{c}
n \\
n-1
\end{array}\right) D(A)-(D(A) P+P D(A)) \\
& -\left(\begin{array}{c}
n-1 \\
n-2
\end{array}\right)\left(A D(P)+D(P) A^{*}\right)-\left(A D(P) P+P D(P) A^{*}\right) \\
& -\left(\begin{array}{c}
n-2 \\
n-3
\end{array}\right) P D(A) P=0
\end{aligned}
$$

and

$$
\begin{aligned}
f_{n-2}(A, P)= & \left(\begin{array}{c}
n \\
n-2
\end{array}\right) D\left(A^{2}\right)-\left(\begin{array}{l}
n-1 \\
n-2
\end{array}\right)\left(D(A) A^{*}+A D(A)\right) \\
& -\left(\begin{array}{l}
n-1 \\
n-3
\end{array}\right)\left(D(P) A^{* 2}+A^{2} D(P)\right) \\
& -\left(\begin{array}{l}
n-2 \\
n-3
\end{array}\right)\left(A D(A) P+P D(A) A^{*}\right)-A D(P) A^{*} \\
& -\left(\begin{array}{l}
n-2 \\
n-4
\end{array}\right) P D\left(A^{2}\right) P=0 .
\end{aligned}
$$

The above equations reduce to

$$
\begin{aligned}
n D(A)= & D(A) P+P D A+(n-1)\left(A D(P)+D(P) A^{*}\right) \\
& +A D(P) P+P D(P) A^{*}+(n-2) P D(A) P
\end{aligned}
$$

and

$$
\begin{aligned}
n(n-1) D\left(A^{2}\right)= & 2(n-1)\left(A D(A)+D(A) A^{*}\right) \\
& +(n-1)(n-2)\left(A^{2} D(P)+D(P) A^{* 2}\right) \\
& +2(n-2)\left(A D(A) P+P D(A) A^{*}\right) \\
& +(n-2)(n-3) P D\left(A^{2}\right) P+2 A D(P) A^{*},
\end{aligned}
$$


respectively. Using (5) the relation (8) reduces to

$$
\begin{aligned}
n(n-1) D\left(A^{2}\right)= & 2(n-1)\left(A D(A)+D(A) A^{*}\right) \\
& +(n-1)(n-2)\left(A^{2} D(P)+D(P) A^{* 2}\right) \\
& +2(n-2)\left(A D(A) P+P D(A) A^{*}\right) \\
& +(n-2)(n-3) P D\left(A^{2}\right) P .
\end{aligned}
$$

Applying the relation (4) and the fact that $A P=P A=A$ and $A^{*} P=P A^{*}=$ $A^{*}$, we have $A D(P) P=A(P D(P) P)=0$ and $P D(P) A^{*}=(P D(P) P) A^{*}=$ 0 . The relation $(7)$ can now be written as

$$
\begin{aligned}
n D(A)= & D(A) P+P D(A)+(n-1)\left(A D(P)+D(P) A^{*}\right) \\
& +(n-2) P D(A) P .
\end{aligned}
$$

Left multiplication of the relation (10) by $P$ gives

$$
P D(A)=A D(P)+P D(A) P .
$$

Similarly, one obtains

$$
D(A) P=D(P) A^{*}+P D(A) P .
$$

Subtracting the relation (11) from the relation (12) yields

$$
D(A) P-P D(A)+A D(P)-D(P) A^{*}=0 .
$$

Using relations (11) and (12) in (10) we obtain

$$
D(A)=A D(P)+D(P) A^{*}+P D(A) P .
$$

Using the relation (14) in the relation (10) gives

$$
2 D(A)=D(A) P+P D(A)+D(P) A^{*}+A D(P) .
$$

Subtracting the relation (14) from the relation (15) gives

$$
D(A)=D(A) P+P D(A)-P D(A) P .
$$

Multiplying the relation (12) from the right side by $A^{*}$ and relation (11) from the left side by $A$, we obtain

$$
D(A) A^{*}=D(P) A^{* 2}+P D(A) A^{*}
$$

and

$$
A D(A)=A^{2} D(P)+A D(A) P .
$$

Combining relations (9), (17) and (18) we obtain

$$
\begin{aligned}
n(n-1) D\left(A^{2}\right)= & n(n-1)\left(A^{2} D(P)+D(P) A^{* 2}\right) \\
& +2(2 n-3)\left(A D(A) P+P D(A) A^{*}\right) \\
& +(n-2)(n-3) P D\left(A^{2}\right) P .
\end{aligned}
$$


Combining relations (17) and (18) we obtain

$$
D(A) A^{*}+A D(A)=D(P) A^{* 2}+A^{2} D(P)+P D(A) A^{*}+A D(A) P .
$$

By comparing (19) and (20) we obtain

$$
\begin{aligned}
& n(n-1) D\left(A^{2}\right)=n(n-1)\left(D(A) A^{*}+A D(A)\right) \\
& \quad+(n-2)(n-3)\left(P D\left(A^{2}\right) P-A D(A) P-P D(A) A^{*}\right) .
\end{aligned}
$$

Using the relations (17) and (18) in the above relation we arrive at

$$
\begin{aligned}
& n(n-1) D\left(A^{2}\right)=n(n-1)\left(D(A) A^{*}+A D(A)\right) \\
& +(n-2)(n-3)\left(P D\left(A^{2}\right) P+D(P) A^{* 2}-D(A) A^{*}+A^{2} D(P)-A D(A)\right) .
\end{aligned}
$$

Putting $A^{2}$ for $A$ in the relation (14) gives

$$
D\left(A^{2}\right)=A^{2} D(P)+D(P) A^{* 2}+P D\left(A^{2}\right) P .
$$

Using the relation (23) in the relation (22) one obtains after some calculation

$$
D\left(A^{2}\right)=D(A) A^{*}+A D(A) .
$$

From the relation (16) one can conclude that $D$ maps $\mathcal{F}(H)$ into itself. We have therefore an additive mapping $D$ which maps $\mathcal{F}(H)$ into itself satisfying the relation (24) for all $A \in \mathcal{F}(H)$. In other words $D$ is a Jordan *-derivation of $\mathcal{F}(H)$. Since all the assumptions of Theorem 1 are fulfilled, one can conclude that there exists a unique $B \in \mathcal{L}(H)$ such that

$$
D(A)=B A^{*}-A B,
$$

holds for all $A \in \mathcal{F}(H)$. It remains to prove that the above relation holds on $A(H)$ as well. Let us introduce $D_{1}: A(H) \rightarrow \mathcal{L}(H)$ by $D_{1}(A)=B A^{*}-$ $A B$ and consider $D_{0}=D-D_{1}$. The mapping $D_{0}$ is, obviously, additive and satisfies the relation (3). Besides $D_{0}$ vanishes on $\mathcal{F}(H)$. Let $A \in A(H)$, let $P \in \mathcal{F}(H)$, be a self-adjoint projection and $S=A+P A P-(A P+P A)$. Since, obviously, $S-A \in \mathcal{F}(H)$, we have $D_{0}(S)=D_{0}(A)$. Besides $S P=P S=0$ and $S^{*} P=P S^{*}$. We have therefore

$$
D_{0}\left(A^{n}\right)=D_{0}(A) A^{* n-1}+A D_{0}\left(A^{n-2}\right) A^{*}+A^{n-1} D_{0}(A)
$$


for all $A \in A(H)$. Applying the above relation we obtain

$$
\begin{aligned}
D_{0}(S) & S^{* n-1}+S D_{0}\left(S^{n-2}\right) S^{*}+S^{n-1} D_{0}(S)=D_{0}\left(S^{n}\right) \\
= & D_{0}\left(S^{n}+P\right)=D_{0}\left((S+P)^{n}\right) \\
= & D_{0}(S+P)(S+P)^{* n-1}+(S+P) D_{0}\left((S+P)^{n-2}\right)(S+P)^{*} \\
& +(S+P)^{n-1} D_{0}(S+P) \\
= & D_{0}(S)\left(S^{* n-1}+P\right)+(S+P) D_{0}\left(S^{n-2}\right)\left(S^{*}+P\right) \\
& +\left(S^{n-1}+P\right) D_{0}(S) \\
= & D_{0}(S) S^{* n-1}+D_{0}(S) P+S D_{0}\left(S^{n-2}\right) S^{*}+S D_{0}\left(S^{n-2}\right) P \\
& +P D_{0}\left(S^{n-2}\right) S^{*}+P D_{0}\left(S^{n-2}\right) P+S^{n-1} D_{0}(S)+P D_{0}(S) .
\end{aligned}
$$

From the above relation it follows that

$$
D_{0}(S) P+S D_{0}\left(S^{n-2}\right) P+P D_{0}\left(S^{n-2}\right) S^{*}+P D_{0}\left(S^{n-2}\right) P+P D_{0}(S)=0 .
$$

Since $D_{0}(S)=D_{0}(A)$, we obtain

$$
\begin{gathered}
D_{0}(A) P+S D_{0}\left(A^{n-2}\right) P+P D_{0}\left(A^{n-2}\right) S^{*} \\
+P D_{0}\left(A^{n-2}\right) P+P D_{0}(A)=0 .
\end{gathered}
$$

Two-sided multiplication of the above relation by $P$ gives

$$
2 P D_{0}(A) P+P D_{0}\left(A^{n-2}\right) P=0 .
$$

Putting $2 A$ for $A$ in the above relation, we obtain

$$
2 P D_{0}(A) P+2^{n-3} P D_{0}\left(A^{n-2}\right) P=0 .
$$

Subtracting the relation (26) from the relation (27) gives

$$
P D_{0}\left(A^{n-2}\right) P=0
$$

which means that

$$
P D_{0}(A) P=0
$$

as well. Right multiplication of the relation (25) by $P$ and using the relations (28) and (29) give

$$
D_{0}(A) P+S D_{0}\left(A^{n-2}\right) P=0 .
$$

Putting $2 A$ for $A$ in the above relation, we obtain (see how the relation (28) was obtained from the relation (26))

$$
D_{0}(A) P=0 .
$$

Since $P$ is an arbitrary one-dimensional projection, it follows from the above relation that $D_{0}(A)=0$ for any $A \in A(H)$. The proof of the theorem is therefore complete. 


\section{REFERENCES}

[1] M. Brešar and J. Vukman, On some additive mappings in rings with involution, Aequationes Math. 38 (1989), 178-185.

[2] M. Brešar and B. Zalar, On the structure of Jordan ${ }^{*}$-derivations, Colloq. Math. 63 (1997), 163-171.

[3] H. G. Dales, Automatic continuity: a survey, Bull. Lond. Math. Soc. 10 (1978), 129-183.

[4] M. G. Dales, Banach algebras and automatic continuity, Calderon Press, Oxford, 2001.

[5] D. Ilišević, Equations arising from Jordan *-derivation pairs, Aequationes Math. 67 (2004), 236-240.

[6] D. Ilišević, On quadratic functionals from * -rings to a class of Banach ${ }^{*}$-algebras, in Proceedings of the Postgraduate School and Conference Functional Analysis VIII, Aarhus Univ., Aarhus, 2004, 110-115.

[7] D. Ilišević, Quadratic functionals on modules over ${ }^{*}$-rings, Studia Sci. Math. Hungar. 42 (2005), 95-105.

[8] D. Ilišević, Quadratic functionals on modules over alternative *-rings, Studia Sci. Math. Hungar. 42 (2005), 459-469.

[9] S. Kurepa, The Cauchy functional equation and scalar product in vector spaces, Glasnik Mat. Fiz.-Astronom. Ser. II Društvo Mat. Fiz. Hrvatske 19 (1964), 23-26.

[10] S. Kurepa, Quadratic and sesquilinear functionals, Glasnik Mat. Fiz.-Astronom. Ser. II Društvo Mat. Fiz. Hrvatske 20 (1965), 79-92.

[11] L. Molnár, Jordan ${ }^{*}$-derivation pairs on a complex ${ }^{*}$-algebras, Aequationes Math. 54 (1997), 44-55.

[12] A. M. Sinclair, Automatic continuity of linear operators, Cambridge University Press, Cambridge, London, New York and Melbourne, 1976.

[13] P. Šemrl, On quadratic functionals, Bull. Aust. Math. Soc. 37 (1988), 27-28.

[14] P. Šemrl, Quadratic and quasi-quadratic functionals, Proc. Amer. Math. Soc. 119 (1993), 1105-1113.

[15] P. Šemrl, Jordan *-derivations of standard operator algebras, Proc. Amer. Math. Soc. 120 (1994), 515-518

[16] P. Vrbová, Quadratic functionals and bilinear forms, Časopis Pest. Mat. 98 (1973), 159-161.

[17] J. Vukman, A result concerning additive functions in hermitian Banach* *algebras and an application, Proc. Amer. Math. Soc. 91 (1984), 367-372.

[18] J. Vukman, Some results concerning the Cauchy functional equation in certain Banach algebras, Bull. Aust. Math. Soc. 31 (1985), 137-144.

[19] J. Vukman, Some functional equations in Banach algebras and an application, Proc. Amer. Math. Soc. 100 (1987), 133-136.

[20] J. Vukman, A note on Jordan *-derivations in semiprime rings with involution, Int. Math. Forum 1 (2006), 617-622.

[21] D. Yang, Jordan *-derivation pairs on standard operator algebras and related results, Colloq. Math. 102 (2005), 137-145.

[22] B. Zalar, Jordan-von Neumann theorem for Saworotnow's generalized Hilbert space, Acta Math. Hungar. 69 (1995), 301-325.

[23] B. Zalar, Jordan *-derivation pairs and quadratic functionals on modules over *rings, Aequationes Math. 54 (1997), 31-43. 
I. Kosi-Ulbl

Faculty of Mechanical Engineering

University of Maribor

Smetanova 17, 2000 Maribor

Slovenia

E-mail: irena.kosi@um.si

J. Vukman

Koroška cesta 57, 2000 Maribor

Slovenia

E-mail: joso.vukman@guest.um.si

Received: 10.9.2014. 